\title{
The Protect Role of Serum Uric Acid to Premature Rupture of Membranes in Gestational Diabetes: a Cross-sectional Study
}

\author{
Meixiang Guo \\ Shanghai Fengxian Central Hospital \\ Jun Lu \\ Shanghai Fengxian Central Hospital \\ Xiaowen $\mathrm{Hu}$ \\ Jinan Central Hospital Affiliated to Shandong University \\ Wenjing Hou \\ Shanghai Fengxian Central Hospital \\ Xuemei Yu \\ Shanghai Fengxian Central Hospital \\ Shuguang Pang ( $\sim 15318816292 @ 163 . c o m$ ) \\ Jinan Central Hospital Affiliated to Shandong University
}

\section{Research article}

Keywords: Uric acid, premature rupture of fetal membranes, Gestational diabetes

Posted Date: December 16th, 2020

DOI: https://doi.org/10.21203/rs.3.rs-126245/v1

License: (c) (i) This work is licensed under a Creative Commons Attribution 4.0 International License. Read Full License 


\section{Abstract}

Background: Uric acid has strong antioxidant activity whereas its oxidative damage is closely related to many diseases. We assessed the association between serum uric acid (SUA) levels and premature rupture of membranes (PROM) in pregnant women with Gestational diabetes (GDM) in china.

Method: In this cross-sectional study, a total of 456 pregnant women were enrolled. Anthropometric parameters for pregnant women were collected within 12 weeks of gestation. Weight gain during pregnancy was obtained from patients' record. GDM was diagnosed according to 75-g oral glucose tolerance tests at 24-28th week's gestation, and SUA was determined simultaneously. PROM was identified as the natural rupture of fetal membranes before the first stage of labor. Logistic models were fitted to identify the presence of PROM using clinical characteristics with (Model 1) or without serum uric acid (Model 2).

Results: There exist differences in BMI, haemoglobin A1c, fasting blood glucose, 1-hour postprandial glucose, $2 \mathrm{~h} \mathrm{PG}$, insulin levels, weight gain during pregnancy, the rate of macrosomia, PROM between women with and without GDM (all P<0.05). Furthermore, individuals with PROM had lower level of SUA than those without PROM in GDM women $(P=0.030)$. The odds ratio of PROM decreases with the increase of SUA level. The area under the receiver operating characteristic curves for PROM based on Model 1 was larger than that in Model 2 ( 0.86 versus $0.71, p<0.05)$.

Conclusion: Elevated relatively SUA levels at the 24-28th weeks of gestation were associated with a lower risk of PROM in women with GDM. SUA may be a protective factor for PROM in GDM patient. The optimal concentration of uric acid in different diseases and different populations needs to be further studied.

\section{Introduction}

Premature rupture of the membranes (PROM) is when rupture of the fetal membranes occurs in pregnancy at any time before the onset of uterine contractions and occurs in around 5 to $10 \%$ of pregnancies. This sometimes occurs prior to 37 weeks of gestation and is then referred to as preterm PROM [1]. PROM is not only a common obstetrics complication but also the leading cause of perinatal mortality and morbidity [2]. PROM is the major determinant of survival and the length of stay for newborns in the neonatal intensive care unit [3]. Additionally, this complication is a common reason for sub-par health in neonates [4], and it is harmful to newborns health in many ways[5]. PROM is also one of the most common maternal complications in women with gestational diabetes (GDM)[2].

GDM affects around 6\% of pregnant women [6]. The International Association of Diabetes and Pregnancy Study Groups (IADPSG) define GDM as is defined as "any degree of glucose intolerance with onset or first recognition during pregnancy" [7]. After a new family planning policy was put into effect in China, the number of women with advanced maternal age increased; and this had an influence on the number of women with GDM; the incidence of GDM increased and so has the risk of adverse perinatal outcomes [8]. 
An important aspect of GDM is increased levels of oxidative stress (OS) biomarkers. Study suggests that OS may affect the pregnancy outcome of GDM patients[9]. Placental histological examinations have indicated that $\mathrm{OS}$ also plays an important role in inducing inflammation and impairing perfusion in the placenta [10]. OS-induced damage affects not only intrauterine tissues and fetal membranes of the placenta but also all major cellular factors of the fetal cells; this damage promotes fetal cell ageing [11]. After receiving a signal for disease or normal maturation, the fetus produces $V$ collagenase, which destroys the basement membrane and the fetal membrane function [12]. It is not clear whether factors related to OS have a relationship to PROM in women with GDM. Although PROM is common in women with GDM, as far as we know there have been no studies that have identified metabolic risk factors for PROM in women with GDM. Therefore, to address this answered question, we studied the morbidity of PROM in GDM and non-GDM pregnant and explored whether the metabolic risk factors would probably have effect on PROM in the GDM group.

\section{Materials And Methods}

\section{Subjects and grouping}

In this cross-sectional study, the subjects were recruited from the maternity clinic of the Central Hospital of Shanghai Fengxian District from November 2016 to January 2019. The patients were included according to the following criteria: 1) 20 to 40 years old, 2) had a pregnancy examination in our hospital, 3) were prepared to complete a 75-g oral glucose tolerance test (OGTT) screening at $24 \sim 28$ weeks and 4) were due to give birth in our hospital to ensure the integrity of the data. All subjects underwent a medical history evaluation, and those with the following were excluded: 1 ) a history of smoking $(n=1), 2)$ diabetes diagnosis before pregnancy $(n=15), 3)$ polycystic ovary syndrome $(n=15), 4)$ hypertension $(n=$ $8), 5)$ liver disease $(n=3)$, or 6$)$ drug use $(n=5)$. Finally, a total of 152 women with GDM and 304 individuals without GDM were included in this study.

\section{Data collection}

Pre-pregnancy BMI $(\mathrm{kg} / \mathrm{m} 2)$ was calculated using self-report weight before pregnancy divided squared height measured at 12 weeks of gestation.

Venous blood samples were drawn after a minimum of 10-h overnight fast during 24-28 gestational weeks. Serum biochemical parameters were measured using a Beckman DXC800 automatic biochemical analyzer. Haemoglobin A1c (HbA1c) levels were measured using high-performance liquid chromatography (TOSOH HLC-723 G7, Japan), and insulin levels were determined using an insulin detection kit and its reagent (Roche Diagnostics, Germany).

One-step $75 \mathrm{~g}$ OGTTs were performed during 24-28 gestational weeks. Fasting and post-load blood glucose and insulin levels were measured at 0,60 and $120 \mathrm{~min}$. GDM were diagnosed according to the 2013 World Health Organization diagnostic criteria: a fasting blood glucose $\geq 5.1 \mathrm{mmol} / \mathrm{L}$ or a 1-hour OGTT blood glucose $\geq 10.0 \mathrm{mmol} / \mathrm{L}$, or a 2 -hour OGTT blood glucose $\geq 8.5 \mathrm{mmol} / \mathrm{L}$. 
All participants were inpatients before delivery. Weight gain during pregnancy were collected and primary adverse outcomes were confirmed by experience obstetrician. The natural rupture of fetal membranes before the first stage of labor was diagnosed as PROM. All subjects had been delivered and finished follow-up in December 2019.

\section{Statistical analysis}

Continuous data were tested for normal distribution using the Kolmogorov-Smirnov test. Normally distributed continuous data are presented as means \pm standard deviation and a t-test was used for comparison. Non-normally distributed continuous data are presented as medians (range) and a MannWhitney $\mathrm{U}$ test was used for comparison. Categorical variables were expressed as frequencies (percentage) and a Chi-square test was used for comparison. The blood uric acid level of the total population is divided into three groups according to the tertiles, The association between SUA level and PROM was assessed using binary logistic regression, odds ratios (ORs) and $95 \%$ confidence intervals $(95 \% \mathrm{Cls})$ were presented; The predictive value of different models for PROM was analysed using the receiver operating characteristic (ROC) analysis. Statistical analyses were performed using SPSS for Windows version 20.0 (IBM SPSS, New York, United States). Two-sided p-values $<0.05$ were considered statistically significant.

\section{Results}

\section{Comparison of clinical characteristics and outcomes}

Table 1 lists the clinical characteristics of the patients involved in this study. There existed difference in levels of HBA1c, FPG, 1-hour postprandial glucose, 2 h PG, 2-h INS, HOMA-IR, HOMA- $\beta$, white blood cells, $B M I$, fasting insulin, 1-hour INS and triglycerides between the non-GDM and GDM groups (all $P<0.05$ ). Moreover, there were significant differences in weight gain during pregnancy $(P=0.014)$, fetus birth weight $(P=0.003)$, rate of PROM $(P=0.033)$ and macrosomia $(P=0.004)$ between the two groups. There was similar level of systolic blood pressure before delivery in the two groups. 
Table 1

clinical characteristics and main outcome in non-GDM and GDM groups

\begin{tabular}{|c|c|c|c|}
\hline \multirow[t]{2}{*}{ Variable } & \multirow{2}{*}{$\begin{array}{l}\text { non-GDM group } \\
(n=304)\end{array}$} & \multirow{2}{*}{$\begin{array}{l}\text { GDM group } \\
(n=152)\end{array}$} & \multirow[t]{2}{*}{ P-value } \\
\hline & & & \\
\hline Age (years) & $29(26-32)$ & $29(26-33)$ & 0.171 \\
\hline Pre-pregnancy BMI (kg/m2) & $21.2(19.6-23.2)$ & $22.3(20.2-24.2)$ & $0.001 *$ \\
\hline Systolic blood pressure (mmHg) & 110(104-120) & $112(102-120)$ & 0.294 \\
\hline Diastolic blood pressure $(\mathrm{mmHg})$ & $70(65-75)$ & $70(65-78)$ & 0.673 \\
\hline HbA1c (\%) & $4.80(4.50-4.90)$ & $4.90(4.40-5.20)$ & $<0.001 *$ \\
\hline FPG (mmol/L) & $4.20(4.00-4.50)$ & $4.90(4.40-5.65)$ & $<0.001 *$ \\
\hline 1-h PG (mmol/L) & $6.70(5.70-7.95)$ & $9.80(7.78-10.90)$ & $<0.001^{*}$ \\
\hline 2-h PG (mmol/L) & $6.30(5.40-6.90)$ & $8.50(6.13-9.20)$ & $<0.001^{*}$ \\
\hline FINS (mU/L) & $8.72(5.96-13.0)$ & $10.3(6.7-15.5)$ & 0.010 * \\
\hline 1-h INS (mU/L) & $61.4(42.5-86.4)$ & $74.0(48-117)$ & 0.010 * \\
\hline 2-h INS (mU/L) & $60.7(39.8-82.7)$ & 84.06(51.9-133.) & $<0.001 *$ \\
\hline $\operatorname{ALT}(\mathrm{U} / \mathrm{L})$ & $15(11-23)$ & $13(10-22)$ & 0.30 \\
\hline Total bilirubin $(\mu \mathrm{mol} / \mathrm{L})$ & $8.0(6.9-10.0)$ & $8.00(6.25-9.69)$ & 0.57 \\
\hline Creatinine(umolL) & $46(43-51)$ & $47(42-51)$ & 0.49 \\
\hline Uric acid ( $\mu \mathrm{mol} / \mathrm{L})$ & 213(184-259) & $230(195-256)$ & 0.130 \\
\hline Triglycerides (mmol/L) & $2.17(1.73-2.80)$ & $2.41(1.90-3.11)$ & $0.023^{*}$ \\
\hline Total cholesterol (mmol/L) & $6.07(5.53-6.82)$ & $5.97(5.27-6.66)$ & 0.093 \\
\hline White blood cell $(\times 109 / L)$ & $9.12 \pm 2.02$ & $9.90 \pm 2.07$ & $<0.001^{*}$ \\
\hline Platelet count $(\times 109 / L)$ & $218 \pm 48$ & $224 \pm 42$ & 0.06 \\
\hline Parity & $2(1-2)$ & $1(1-2)$ & 0.44 \\
\hline Weight gain during pregnancy $(\mathrm{Kg})$ & $15.9 \pm 5.37$ & $14.1 \pm 6.5$ & $0.014^{*}$ \\
\hline Fetus birth weight (g) & $3399 \pm 462$ & $3542 \pm 448$ & $0.003^{*}$ \\
\hline \multicolumn{4}{|c|}{$\begin{array}{l}\text { Data were expressed as median(interquartile range) or mean } \pm \text { standard deviation, Significant at } P< \\
\text { 0.05. } P<0.05^{\star}\end{array}$} \\
\hline \multicolumn{4}{|c|}{$\begin{array}{l}\text { Abbreviation: PROM : premature rupture of the membranes BMI: body mass index; HbA1c: } \\
\text { hemoglobin A1C; FPG: fasting plasma glucose; 1-h PG: 1-hour postprandial glucose; 2-h PG: 2-hour } \\
\text { postprandial glucose; FINS: fasting insulin; 1-h INS: 1-hour postprandial insulin; 2-h INS: 2-hour } \\
\text { postprandial insulin; ALT: alanine aminotransferase. }\end{array}$} \\
\hline
\end{tabular}




\begin{tabular}{|c|c|c|c|}
\hline \multirow[t]{2}{*}{ Variable } & non-GDM group & GDM group & \multirow[t]{2}{*}{ P-value } \\
\hline & $(n=304)$ & $(n=152)$ & \\
\hline PROM (n,\%) & $22(7.6)$ & 19(13.29) & $0.033^{*}$ \\
\hline Macrosomia(n,\%) & $20(6.99)$ & 24(16.78) & $0.004 *$ \\
\hline Fetal distress in the uterus $(n, \%)$ & $25(8.74)$ & $13(0.091)$ & 0.991 \\
\hline \multicolumn{4}{|c|}{$\begin{array}{l}\text { Data were expressed as median(interquartile range) or mean } \pm \text { standard deviation, Significant at } P< \\
0.05 . P<0.05^{*}\end{array}$} \\
\hline \multicolumn{4}{|c|}{$\begin{array}{l}\text { Abbreviation: PROM : premature rupture of the membranes BMI: body mass index; HbA1c: } \\
\text { hemoglobin A1C; FPG: fasting plasma glucose; 1-h PG: 1-hour postprandial glucose; 2-h PG: 2-hour } \\
\text { postprandial glucose; FINS: fasting insulin; 1-h INS: 1-hour postprandial insulin; 2-h INS: 2-hour } \\
\text { postprandial insulin; ALT: alanine aminotransferase. }\end{array}$} \\
\hline
\end{tabular}

\section{Clinical characteristics in women with and without PROM in GDM group}

The patients with GDM were divided into PROM and non-PROM groups and the baseline data for the two sub-groups were shown in Table 2. There were significant differences in age $(P=0.030)$ and uric acid $(P=$ 0.030 ) between the two groups. There were no significant difference in uric acid between GDM and nonGDM group when regardless of PROM status $(P=0.130)$, Table 1. 
Anthropometric parameters and biochemical indexes in the PROM group and non-PROM sub-groups in the GDM group

\begin{tabular}{|c|c|c|c|}
\hline \multirow[t]{2}{*}{ Variable } & non-PROM group & PROM group & \multirow[t]{2}{*}{ P-value } \\
\hline & $(n=130)$ & $(n=22)$ & \\
\hline Age (years) & $29 \rrbracket 26 \rrbracket 32 \rrbracket$ & $32 \llbracket 28 \bowtie 34 \rrbracket$ & $0.030 *$ \\
\hline Pre-pregnancy BMI (kg/m2) & $22.21 \rrbracket 20.20 \rrbracket 24.72 \rrbracket$ & $22.57 \rrbracket 20.22 \rrbracket 24.45 \rrbracket$ & 0.778 \\
\hline $\mathrm{SBP}(\mathrm{mmHg})$ & $114.00 \rrbracket 106.50 \rrbracket 120.00 \rrbracket$ & $112.00 \rrbracket 100.00 \rrbracket 121.25 \rrbracket$ & 0.676 \\
\hline $\mathrm{DBP}(\mathrm{mmHg})$ & $70.00 \rrbracket 65.00 \rrbracket 80.00 \rrbracket$ & $70.00 \rrbracket 65.25 \rrbracket 75.00 \rrbracket$ & 0.478 \\
\hline HbA1c (\%) & $4.90 \rrbracket 4.30 \rrbracket 5.50 \rrbracket$ & $4.90 \rrbracket 4.70 \rrbracket 5.10 \rrbracket$ & 0.839 \\
\hline FPG (mmol/L) & $4.80 \rrbracket 4.30 \rrbracket 5.50 \rrbracket$ & $4.80 \rrbracket 4.50 \rrbracket 5.40 \rrbracket$ & 0.746 \\
\hline 1-h PG (mmol/L) & $9.80 \rrbracket 8.10 \rrbracket 10.90 \rrbracket$ & 10.10ه7.90冈10.90》 & 0.676 \\
\hline 2-h PG (mmol/L) & $8.30 \rrbracket 6.15 \rrbracket 9.15 \rrbracket$ & $9.05 \varangle 6.98 \varangle 9.93 \rrbracket$ & 0.140 \\
\hline FINS (mU/L) & $10.00 \rrbracket 6.64 \rrbracket 13.03 \rrbracket$ & $11.00 \rrbracket 5.30 \rrbracket 21.00 \rrbracket$ & 0.494 \\
\hline 1-h INS (mU/L) & 76.97囚47.80冈119.28囚 & $72.15 \llbracket 40.50 \rrbracket 87.80 \rrbracket$ & 0.444 \\
\hline 2-h INS (mU/L) & $81.30 \rrbracket 51.88 \rrbracket 130.30 \rrbracket$ & $77.00 \rrbracket 49.41 \rrbracket 122.35 \rrbracket$ & 0.579 \\
\hline ALT (U/L) & $12 \rrbracket 10 \bigotimes 21 \rrbracket$ & 11ه9凶14ه & 0.086 \\
\hline Total bilirubin $(\mu \mathrm{mol} / \mathrm{L})$ & $7.70 \rrbracket 6.20 \rrbracket 9.60 \rrbracket$ & $8.00 \rrbracket 6.00 \rrbracket 10.50 \rrbracket$ & 0.923 \\
\hline Creatinine(umolL) & $46 \rrbracket 43 \rrbracket 51 \rrbracket$ & $49 \llbracket 41 \rrbracket 52 \rrbracket$ & 0.668 \\
\hline Uric acid $(\mu \mathrm{mol} / \mathrm{L})$ & $233 \llbracket 203 \rrbracket 255 \rrbracket$ & 197囚187囚237》 & $0.030 *$ \\
\hline Triglycerides (mmol/L) & $2.41 \rrbracket 1.90 \rrbracket 3.05 \rrbracket$ & $2.84 \llbracket 2.03 \llbracket 4.04 \rrbracket$ & 0.146 \\
\hline Total cholesterol (mmol/L) & $5.95 \llbracket 5.24 \rrbracket 6.59 \rrbracket$ & $6.03 \rrbracket 5.44 \llbracket 7.28 \rrbracket$ & 0.353 \\
\hline White blood cell $(\times 109 / L)$ & $9.98 \pm 2.11$ & $10.04 \pm 2.39$ & 0.974 \\
\hline Platelet count $(\times 109 / \mathrm{L})$ & $226.96 \pm 47.22$ & $210.07 \pm 48.49$ & 0.155 \\
\hline Weight gain $₫ \mathrm{Kg} \rrbracket$ & $14.50 \pm 6.50$ & $12.78 \pm 5.34$ & 0.348 \\
\hline Fetus birth weight(g) & $3545.95 \pm 440.31$ & $3510.26 \pm 514.52$ & 0.859 \\
\hline \multicolumn{4}{|c|}{$\begin{array}{l}\text { Data were expressed as median(interquartile range) or mean } \pm \text { standard deviation, Significant at } \\
P<0.05 . P<0.05^{\star}\end{array}$} \\
\hline \multicolumn{4}{|c|}{$\begin{array}{l}\text { Abbreviation: PROM : premature rupture of the membranes BMI: body mass index; HbA1c: } \\
\text { hemoglobin A1C; FPG: fasting plasma glucose; 1-h PG: 1-hour postprandial glucose; 2-h PG: 2-hour } \\
\text { postprandial glucose; FINS: fasting insulin; 1-h INS: 1-hour postprandial insulin; 2-h INS: 2-hour } \\
\text { postprandial insulin; ALT: alanine aminotransferase. }\end{array}$} \\
\hline
\end{tabular}




\section{Correlation between SUA and PROM}

Binary logistic regression was conducted to assess the association of PROM with clinical variables with PROM as the dependent variable, as shown in Table 3. When the clinical variables (age, pre-pregnancy BMI,1-h PG, parity, weight gain) that might be affect outcomes were adjusted, Uric acid $(P=0.043)$ were related to PROM in GDM group in multivariate analysis. Furthermore, the uric acid of the whole population was stratified by three quantile, the result showed the incidence of PROM decreases with the increase of blood uric acid tertiles, the corresponding ORs (95Cls) were $(0.13$ [0.02-0.95]) in the second tertile and $(0.07$ [0.01-0.93], all $\mathrm{P}<0.05)$ in the upper tertile in comparison with lower tertile.

Table 3

Multivariate logistic analysis of factors related to PROM in the non-GDM and GDM group

\begin{tabular}{|lllll|}
\hline & non-GDM & & GDM & \\
\hline variables & OR(95\%Cl) & p-value & OR(95\%Cl) & p-value \\
\hline Age(years) & $1.02(0.84-1.22)$ & 0.901 & $1.19(1.01-1.42)$ & $0.049^{*}$ \\
\hline Pre-pregnancy BMI(kg/m2) & $1.01(0.77-1.30)$ & 0.984 & $1.18(0.84-1.66)$ & 0.345 \\
\hline 1-h PG(mmol/L) & $1.17(0.71-1.91)$ & 0.544 & $1.01(0.66-1.54)$ & 0.963 \\
\hline Parity & $0.75(0.18-3.15)$ & 0.689 & $0.35(0.05-2.23)$ & 0.265 \\
\hline Weight gain(Kg) & $0.94(0.84-1.06)$ & 0.331 & $0.93(0.79-1.09)$ & 0.344 \\
\hline uric acid levels(umol/L) & & 0.579 & & $0.043^{*}$ \\
\hline SCR tertiles & & & & \\
\hline 0-197 & 1 & & 1 & $0.044^{*}$ \\
\hline 198-247 & $2.53(0.36-17.90)$ & 0.352 & $0.13(0.02-0.95)$ & $0.044^{*}$ \\
\hline$\geq 247$ & $2.34(0.43-12.84)$ & 0.329 & $0.07(0.01-0.93)$ & \\
\hline OR: odds ratio; Cl: confidence interval; Significant at $P<0.05 . P<0.05^{*}$ & \\
\hline Abbreviation: 1-h PG: 1 -hour postprandial glucose & & \\
\hline
\end{tabular}

Furthermore, the predictive value of different models for PROM was analysed using the receiver operating characteristic (ROC) analysis. As shown in Fig. 2, ROC curve analysis showed that the area under the curve of general clinical characteristic model 1 was $(0.71$ [0.54-0.87], $p<0.05)$, When uric acid is added as a variable, the area under the curve in model 2 reached $(0.86[0.75-0.98], p<0.001)$.

\section{Discussion}


The present study indicated that women with PROM had lower levels of SUA than those without PROM, and the prevalence of PROM decreased with the increase of SUA tertiles among GDM group. Secondly, multiple logistic regression analysis indicated that SUA was independently associated with PROM in GDM group. Finally, AUC was larger in model incorporating SUA combined with general clinical parameters than that only considered general indices alone.

\section{Main risk factors affected PROM has known in PROM}

What we already known about PROM mainly focused on infectious factors and external factors, such as genital infections by chlamydia and/or gonorrhoea, intrauterine infection and cervical abnormalities before pregnancy [13]. Studies that have looked for metabolic factors related to PROM in pregnant women are also rare. One study from a hospital in Ethiopia showed that a history of abortion, history of PROM, history of caesarean section, and abnormal vaginal discharge were positively associated with PROM [1]. A Chinese study suggested migration as a result of urbanization, high rates of induced abortion, and preterm birth are potential risk factors for preterm PROM [[14]]. While a Swedish study of PROM at or near term found no obvious risk factors and concluded that PROM close to term can be difficult to manage [15]. GDM is associated with adverse pregnancy outcomes including PROM[7]. A study from Pennsylvania found cord blood uric acid was higher in preterm PROM vs preterm birth, but maternal plasma uric acid and glucose tolerance state was not be considered[16]. As far as we know the relationship of uric acid levels with PROM in women with GDM have not been studied previously. No model for individual prediction of the risk of PROM has been validated and their use in clinical practice. By distinguishing between GDM and women with normal glucose tolerance we identified SUA as an independent factor related to PROM for women with GDM. Of utmost importance is that, as the concentration of uric acid increases, the risk of PROM decreases in the patients with GDM, elevated SUA levels suggesting lower risk incidence of PROM, after adjustment for other confounders.

\section{The importance of reasonable uric acid level in GDM}

The concentration of serum uric acid has two sides in the pathophysiological process of many diseases. Hyperglycaemia accelerates purine nucleotide synthesis, which in turn stimulates nucleotide breakdown and increases the concentration of nucleotide degradation products, including superoxide molecules and uric acid [17]. These substances directly damage islet function [18] and worsen diabetes complications[19]. However, uric acid itself is an antioxidant that can protect cells from OS damage. The relationship between uric acid and cardiovascular event rates is J-shaped[20]. High SUA levels due to uric acid toxicity increase cardiovascular events, but when SUA levels are low, cardiovascular events are also increased; this analogous finding was observed in patients with vasospastic angina [21]. The effects of SUA are also the same in acute ischemic stroke patients [22].

Reference values for pregnancy can be extremely important for making clinical decisions. Normal reference values are usually based on blood samples from non-pregnant women. However, uric acid levels are higher in women in late pregnancy than in non-pregnant women [23]. Additionally, hyperglycaemia increases oxidative stress products, so SUA levels should also be increased in GDM 
patients. Women with GDM should have higher SUA levels in late pregnancy and in hyperglycaemic states. However, when the SUA levels do not increase correspondingly, women with GDM are more prone to PROM.

\section{The possible mechanism of uric acid, oxidative stress and PROM}

A general health screening study of individuals revealed that SUA levels are positively associated with antioxidant potential; these results indicate that SUA can prevent oxidative damage[24]. GDM is characterized by increased levels of OS biomarkers. Placental histological examinations have indicated that OS also plays an important role in inducing inflammation and impairing perfusion in the placenta [10]. Our study showed that SUA levels were inversely correlated with PROM in GDM subjects. OS damage is also an important factor in the initiation of cell apoptosis. GDM and type 2 diabetes mellitus (T2DM) subjects have increased expression levels of base excision repair proteins and hyperglycaemia levels are associated with apoptosis [25]. Proteomics research confirmed by western blot and RT-PCR analyses revealed that placental tissues from PROM subjects had lower levels of the anti-apoptotic protein HSPA2 and higher expression levels of PRDX3 and annexin A1 (associated with apoptosis) than placental tissues from normal pregnancy subjects[26]. The increase in apoptosis caused by the decrease in antioxidant stress may be another important cause of PROM. We hypothesized that the antioxidant capacity of relatively low SUA levels cannot exert its usual protective effects and thus women with low SUA are at risk of PROM. This study indicates that maternal age is another important factor affecting on PROM, as it was significantly higher in GDM patients with PROM. This is consistent with the Chinese study of risk factors of PROM [14]. The percentage decrease in enzymatic antioxidants and antioxidant vitamins with age is significant [27]. After the implementation of the nationwide two-child policy in China, the amount of elderly pregnant women increased, this may be another important factor in association with adverse pregnancy outcomes.

There was some strength in this study. Blood biochemical tests (including SUA) collected from all subjects at 24-28 weeks of gestation to exclude the effects of differences in gestational weeks on the results. All the subjects were women because of pregnancy, none of the subjects consumed alcohol. Consequently, the possible SUA-influencing factors, such as sex, and alcohol consumption, were excluded. Except for the factors that may affect the glucose metabolism, there were no other recruitment restrictions, so the study population should be a good representation. There are some limitations of this study. This was a single center study, so the number of subjects was limited. The follow-up period was short so we could make no conclusions on the long-term pregnancy outcomes. The related uric acid metabolism pathways were needed further study.

\section{Conclusion}

In conclusion, even the change of uric acid level in physiological concentration can bring adverse effects on PROM and maternal age is another important factor affecting only in gestational diabetes pregnant 
women. The moderate increase of uric acid level in 24-28 gestation may have a potential protective effect on PROM with GDM. At present, we know that the concentration of uric acid is different in different genders and physiological states, and the influence of uric acid on disease outcomes in different diseases is worthy of further study.

\section{Abbreviations}

SUA, serum uric acid; PROM, premature rupture of membranes; IADPSG, International Association of Diabetes and Pregnancy Study Groups ; GDM , gestational diabetes; BMI, body mass index; OS, oxidative stress; FBG, fasting blood glucose; HbA1c, glycosylated hemoglobin; 1-h PG: 1-hour postprandial glucose; 2-h PG: 2-hour postprandial glucose; FINS: fasting insulin; 1-h INS: 1-hour postprandial insulin; 2-h INS: 2hour postprandial insulin; ALT: alanine aminotransferase.

\section{Declarations}

\section{Statement of Ethics}

The study was approved by the Ethics Committee of the Central Hospital of Fengxian District (Approval number: No. KY201604) and conducted according to the 1995 Declaration of Helsinki (as revised in Fortaleza, Brazil, October 2013). All subjects provided informed consent, and patient anonymity was preserved.

\section{Consent for publication}

Not Applicable.

\section{Availability of data and materials}

The datasets used and/or analysed during the current study are available from the corresponding author on reasonable request.Due to the data containing potentially identifying and sensitive patient information as well as the consent agreement supervised by the ethics committee. Data are available from the Ethics Committee of Central Hospital of Shanghai Fengxian District for researchers who meet the criteria for access to confidential data.

\section{Disclosure Statement}

The authors declare that they have no conflicts of interest associated with this article. No benefits in any form have been received or will be received from a commercial party related directly or indirectly to the subject of this paper.

\section{Funding}


This study was supported by Shandong Province Key R\&D Plan (Grant No. 2016GSF201019), Jinan Science and Technology Innovation Program of Clinical Medicine (Grant No. 201705072) and Academic promotion programme of Shandong First Medical University(Grant No. 2019QL025).

\section{Authors' contributions}

S.P. and X.Y. designed the study; M.G., Jun Lu, W.H., X.H. conducted the research; M.G. and J.L. analyzed the data and wrote the manuscript. The final manuscript was read and approved by all authors.

\section{References}

1. Assefa NE, Berhe H, Girma F, Berhe K, Berhe YZ, Gebreheat G, Werid WM, Berhe A, Rufae HB, Welu G. Risk factors of premature rupture of membranes in public hospitals at Mekele city, Tigray, a case control study. BMC Pregnancy Childbirth. 2018;18:386.

2. Ghosh S, Ghosh K. Maternal and neonatal outcomes in gestational diabetes mellitus. Journal of the Indian Medical Association. 2013;111:330-1, 6.

3. Kurek Eken M, Tuten A, Ozkaya E, Karatekin G, Karateke A. Major determinants of survival and length of stay in the neonatal intensive care unit of newborns from women with premature preterm rupture of membranes. J Matern Fetal Neonatal Med. 2017;30:1972-5.

4. Jie C, Mai S, Qiuju L, Fang Y, Fang L, Qinying F, Yong Z, Lin W. Investigation of sub-health status of neonates in Heping street region (Beijing) and analysis on related risk factors. Pakistan journal of pharmaceutical sciences. 2016;29:2173-7.

5. Wu J, Liu J, Feng ZC, Huang JJ, Wu G. [Influence of premature rupture of membranes on neonatal health]. Zhonghua er ke za zhi = Chinese journal of pediatrics. 2009;47:452-6.

6. Mack LR, Tomich PG. Gestational Diabetes: Diagnosis, Classification, and Clinical Care. Obstet Gynecol Clin North Am. 2017;44:207-17.

7. International Association of D, Pregnancy Study Groups Consensus P, Metzger BE, Gabbe SG, Persson B, Buchanan TA, Catalano PA, Damm P, Dyer AR, Leiva A, Hod M, Kitzmiler JL, Lowe LP, McIntyre HD, Oats JJ, Omori Y, Schmidt MI. International association of diabetes and pregnancy study groups recommendations on the diagnosis and classification of hyperglycemia in pregnancy. Diabetes Care. 2010;33:676-82.

8. Chen Y, Zheng XL, Wu SW, Zhang WY. [Clinic characteristics of women with advanced maternal age and perinatal outcomes]. Zhonghua fu chan ke za zhi. 2017;52:508-13.

9. Zhang C, Yang Y, Chen R, Wei Y, Feng Y, Zheng W, Liao H, Zhang Z. Aberrant expression of oxidative stress related proteins affects the pregnancy outcome of gestational diabetes mellitus patients. American journal of translational research. 2019;11:269-79.

10. Perrone S, Tataranno ML, Negro S, Longini M, Toti MS, Alagna MG, Proietti F, Bazzini F, Toti P, Buonocore G. Placental histological examination and the relationship with oxidative stress in preterm infants. Placenta. 2016;46:72-8. 
11. Menon R. Oxidative stress damage as a detrimental factor in preterm birth pathology. Front Immunol. 2014;5:567.

12. Lee $\mathrm{T}$, Silver $\mathrm{H}$. Etiology and epidemiology of preterm premature rupture of the membranes. Clin Perinatol. 2001;28:721-34.

13. Schmitz T, Sentilhes L, Lorthe E, Gallot D, Madar H, Doret-Dion M, Beucher G, Charlier C, Cazanave C, Delorme P, Garabédian C, Azria E, Tessier V, Sénat MV, Kayem G. Preterm premature rupture of the membranes: Guidelines for clinical practice from the French College of Gynaecologists and Obstetricians (CNGOF). European journal of obstetrics, gynecology, and reproductive biology. 2019;236:1-6.

14. Zhou Q, Zhang W, Xu H, Liang H, Ruan Y, Zhou S, Li X. Risk factors for preterm premature rupture of membranes in Chinese women from urban cities. Int J Gynaecol Obstet. 2014;127:254-9.

15. Ladfors L, Mattsson LA, Eriksson M, Milsom I. Prevalence and risk factors for prelabor rupture of the membranes (PROM) at or near-term in an urban Swedish population. J Perinat Med. 2000;28:491-6.

16. Menon R, Taylor BD. Exploring Inflammatory Mediators in Fetal and Maternal Compartments During Human Parturition. Obstetrics and gynecology. 2019;134:765-73.

17. Law KP, Zhang $\mathrm{H}$. The pathogenesis and pathophysiology of gestational diabetes mellitus: Deductions from a three-part longitudinal metabolomics study in China. Clin Chim Acta. 2017;468:60-70.

18. Jia L, Xing J, Ding Y, Shen Y, Shi X, Ren W, Wan M, Guo J, Zheng S, Liu Y, Liang X, Su D. Hyperuricemia causes pancreatic beta-cell death and dysfunction through NF-kappaB signaling pathway. PLoS One. 2013;8:e78284.

19. Kushiyama A, Tanaka K, Hara S, Kawazu S. Linking uric acid metabolism to diabetic complications. World J Diabetes. 2014;5:787-95.

20. Verdecchia P, Schillaci G, Reboldi G, Santeusanio F, Porcellati C, Brunetti P. Relation between serum uric acid and risk of cardiovascular disease in essential hypertension. The PIUMA study. Hypertension. 2000;36:1072-8.

21. Gwag HB, Yang JH, Park TK, Song YB, Hahn JY, Choi JH, Lee SH, Gwon HC, Choi SH. Uric Acid Level Has a U-shaped Association with Clinical Outcomes in Patients with Vasospastic Angina. Journal of Korean medical science. 2017;32:1275-80.

22. Kawase S, Kowa H, Suto Y, Fukuda H, Kusumi M, Nakayasu H, Nakashima K. Association between Serum Uric Acid Level and Activity of Daily Living in Japanese Patients with Ischemic Stroke. Journal of stroke and cerebrovascular diseases : the official journal of National Stroke Association. 2017;26:1960-5.

23. Edelstam G, Löwbeer C, Kral G, Gustafsson SA, Venge P. New reference values for routine blood samples and human neutrophilic lipocalin during third-trimester pregnancy. Scandinavian journal of clinical and laboratory investigation. 2001;61:583-92.

24. Ishizaka Y, Yamakado M, Toda A, Tani M, Ishizaka N. Relationship between serum uric acid and serum oxidative stress markers in the Japanese general population. Nephron Clin Pract. 
2014;128:49-56.

25. Moreli JB, Santos JH, Lorenzon-Ojea AR, Correa-Silva S, Fortunato RS, Rocha CR, Rudge MV, Damasceno DC, Bevilacqua E, Calderon IM. Hyperglycemia Differentially Affects Maternal and Fetal DNA Integrity and DNA Damage Response. Int J Biol Sci. 2016;12:466-77.

26. Chang A, Zhang Z, Zhang L, Gao Y, Zhang L, Jia L, Cui S, Wang P. Proteomic analysis of preterm premature rupture of membranes in placental tissue. Arch Gynecol Obstet. 2013;288:775-84.

27. Okoduwa SI, Umar IA, Ibrahim S, Bello F, Habila N. Age-dependent alteration of antioxidant defense system in hypertensive and type-2 diabetes patients. J Diabetes Metab Disord. 2015;14:32.

\section{Figures}

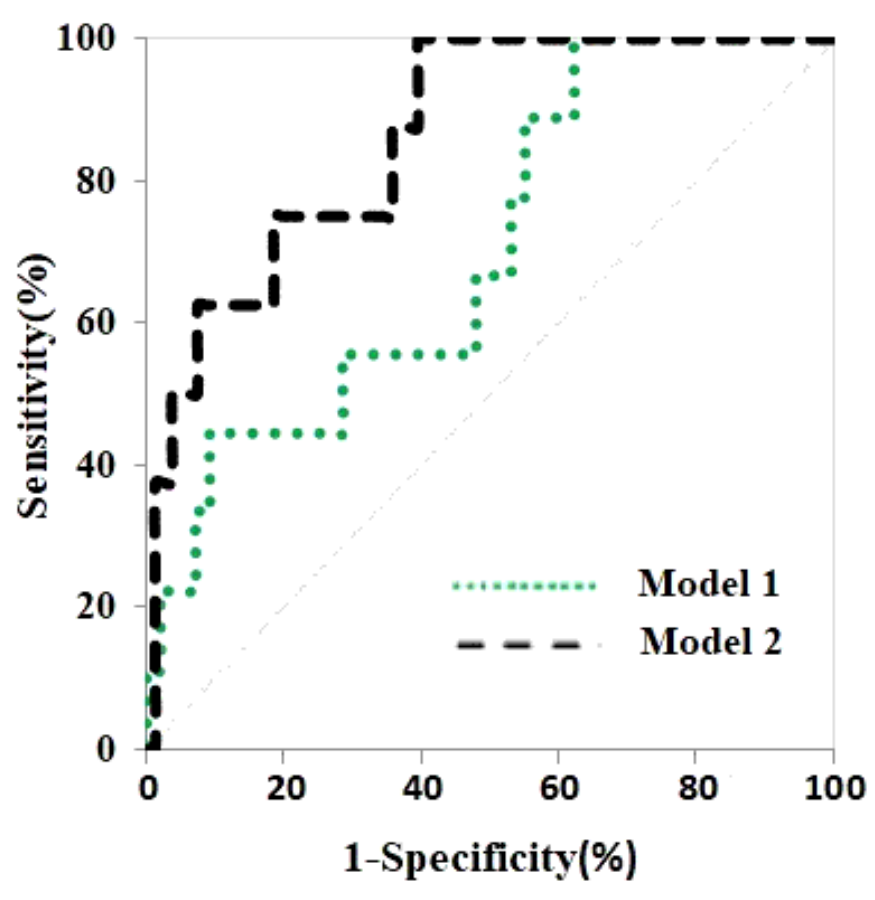

\begin{tabular}{ccc} 
Models & AUCs & P-value \\
\hline Model 1 & $0.71(0.54 \sim 0.87)$ & 0.042 \\
Model 2 & $0.86(0.75 \sim 0.98)$ & 0.001 \\
\hline
\end{tabular}

Model 1: general clinical characteristic model : clinical variables were adjusted (age, pre-pregnancy BMI,1-h PG, parity, weight gain); Model 2: uric acid is added as a variable to the model 1

\section{Figure 1}

ROC curves for SUA predictive of PROM in GDM group. 